\title{
ORIGIN AND DISTRIBUTIONS OF WHITE DWARFS
}

\author{
v. Weidemann \\ Institut $\mathrm{f}$. Theor. Physik und Sternwarte \\ Universität Kiel, Germany (W)
}

Today there is no doubt that white dwarfs represent the most common final stage of stellar evolution. Considerable progress has been made during the last decade in our understanding of their origin and distributions. This is reflected by the fact that it is now possible to predict - from basic theory of stellar evolution and stellar atmospheres - the existence of cooling degenerate stars with nearly the obsexved properties, i.e. a sequence of white dwarfs which fill in their majority a narrow strip in both two-color and colormagnitude diagrams. With other words: the empirically determined surface gravity and radius distributions - which correspond via the mass-radius relation to mass distributions - can now be basically understood within the currently adopted general scheme of stellar evolution with mass loss.

For the first time this opens the possibility of a synthetic approach which I shall adopt in the following. Crude as it may be it nevertheless sets the stage on which we then may post our more detailed questions and judge in which way we may try to answer them.

We therefore assume that stars are born on the main sequence in numbers determined by a time-independent initial mass function (IMF) in a global rate which depends in a defined way on the time in the past, within a specified age of the Galaxy. We let these stars evolve according to currently accepted theory for single stars, through central hydrogen-, shell-, central helium- and double-shell-burning phases until the nuclear evolution is terminated either by fuel exhaustion in a growing degenerate $\mathrm{C} / \mathrm{O}$-core with envelope mass loss or by gravitational collapse and supernova explosion. If we assume the course of this evolution to depend on the initial mass only we arrive at a defined relation between initial and final masses, $\mu_{1 ;}\left(\mu_{i}\right)$. The main sequence birth rate then determines - delayed by the total time of nuclear evolution, and projected by the $\mu_{\mathrm{f}}-\mu_{i}$ relation - the white dwarf (and supernova) birth rate at any chosen epoque of galactic evolution, as well as their mass distribution. This qualitative picture, so far, has been known and accepted for some time, and was first incorporated into a quantitative study of galactic evolution by ostriker and coworkers (Thuan et al., 1975). During the last years, however, it has become evident that mass loss plays a much more important role than anticipated, both steady and unsteady, in stellar winds and/or planetary nebulae (see Reimers, 1975, 
Weldemann 1977a, Wood and Cahn, 1977, Renzin1, 1979). Recent Investigations about white dwarf members in open clusters (Romanishin and Angel, 1979) have shown that progenitor masses for white dwarf production may be as high as $7 / 1$ with a stringent lower limit of $5 \mathrm{\mu} . \mathrm{.}$ The $\mu_{i f-\mu_{i}}$ relation must thus be rather flat, much flatter than assumed by Thuan et al. (1975) whose scheme was based on the unsteady mass loss concept of Paczynski (1970). A wide spectrum of progenitor masses is thus projected into a final mass range which becomes narrower as more low mass main sequence stars are processed compared to higher mass progenitors. This is already secured by the shape of the IMF alone and is even more accentuated if there was higher star productivity in the galactic past. The picture so far is displayed in Fig. 1 - where $J 1_{w}$ marks the limiting mass between white dwarf and supernova progenitors.

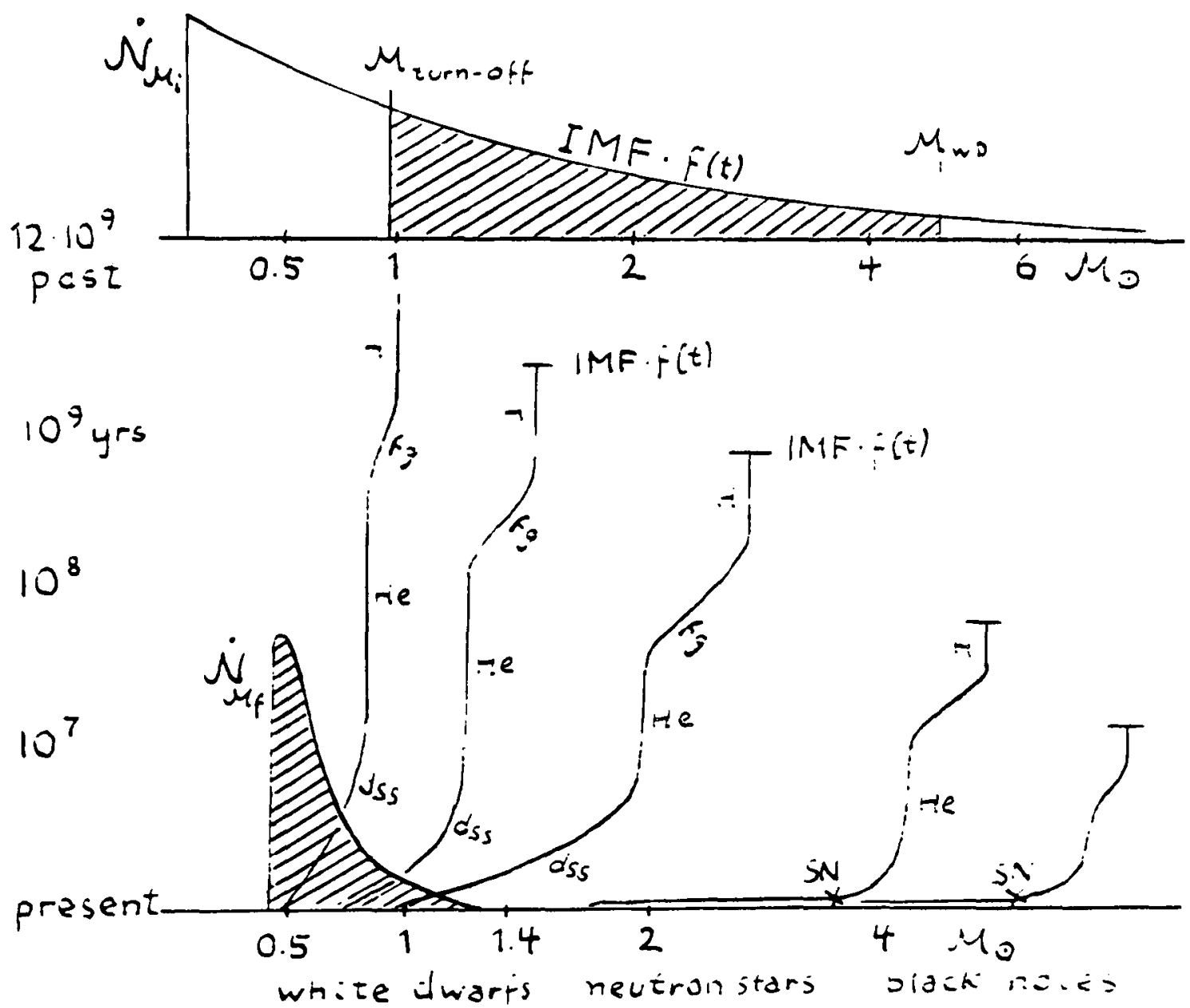

Fig.l Schematic evoluticr. for single stars with wass loss. Birzi rate for iritial masses,,$\therefore$ determined by iritial mass Eurctior, IME, ar: general rate of star formation in the galactic past, E(t). Stars evelve from $\mathrm{B}$ through He burning and lose ass in first giart , fg, an: Eouble shell burning, dss, stage. Upper shaded area aarks mass rarge Iro= which white dwarfs are produced, with super:ovae (SN) bevord a ii=iting mass, $\mathcal{M}_{\mathrm{WD}}$. Lower shaded area: Eresent white dwarf $b i z=-$ zate. 
With white dwarf birth rates thus predicted at any time we may then proceed to use the cooling theory for the calculation of mass distributions in any chosen luminosity interval, and/or of luminosity functions. If the atmospheric composition is specified, we may use the established theory of stellar atmospheres to transform these data into predictions, e.g. of white dwarf distributions in two-color or color-magnitude diagrams.

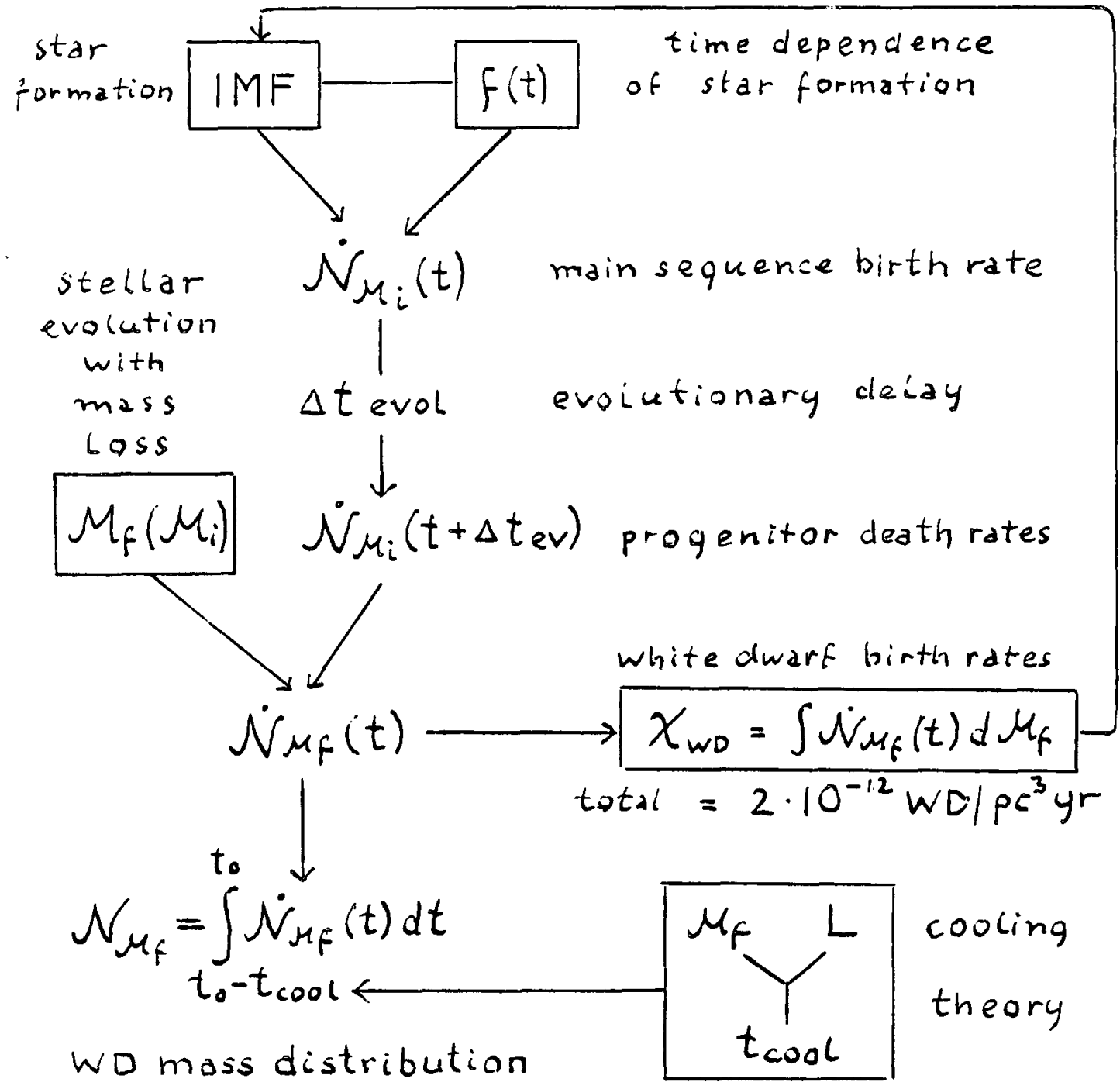

Fig.2 Calculation scheme for white dwarf mass distribution, averaged down to a certain limit of luminosity, $L$, which corresponds for each $M_{f}$ to a certain cooling time, $t_{c 00}$.

The total scheme is presented in Fig. 2. It is synthetic in the sense that it is widely based on theory. It is highly successful in that it predicts the main properties at least for the majority of the observed white dwarfs, the DA stars: a narrow mass distribution as well as the space and velocity aistribution of an intermediate to old stellar population. 
We shall discuss the input data below and first present some results which were obtained in recent calculations in Kiel which followed this scheme. For each choice of an IMF (Salpeter standard,

$\propto \mu^{-2.35} \propto \mu^{-1.5} ; 4$-segment form of Larson and Tinsley 1978; IMF adjusted to reproduce the present luminosity function), of a time dependence of star formation (exponential decline over 5 and 10 billion years, or constant, total age of the model galaxy $12 \cdot 10^{9}$ yrs), and of one of five different relations between initial and final mass,

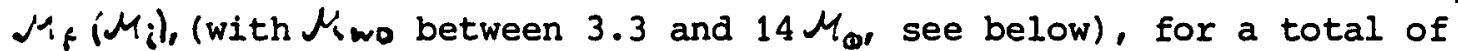
60 models - covering the extremes - we calculated the median mass and mass distributions for the DA range, the fraction of white dwarfs originating from massive progenitors, and the ratio of white dwarf to supernova production. The mean masses varied between 0.53 and $0.91 \mu_{\odot}$, the width of the mass distribution (covering $2 / 3$ of the stars) between 0.07 and $0.58 \mu_{0}$, the fraction of white dwarfs from massive progenitors (more massive than $1.5 \mathcal{M}_{0}$ ) between roughly 25 and $75 \%$, and the ratio of white dwarf to supernova production between 1 and 91 . If white dwarf statistics were sufficiently reliable we thus could put important constraints on models of stellar and galactic evolution. Examples are given in our paper (Koester and Weidemann, 1979). What I do want to emphasize here is the fact that a combined approach promises important clues on the origin of white dwarfs as well as on galactic evolution.

The stage being set we shall now discuss, in turn, our present knowledge of white dwarf statistical data which are relevant within this scheme, the pre-white dwarf stages, especially planetary nebulae, and the question of the initial-final mass relation, especially the limit for white dwarf formation, and then close with some additional remarks.

\section{White dwarf birth rates}

We begin with a number which is in my opinion the best determined as yet: the total white dwarf birth rate in the solar neighborhood at the present epoch of the Galaxy. The method is straightforward: one counts white dwarfs down to a limit of luminosity, corrects the numbers for incompleteness, and divides the numbers by cooling ages to the corresponding luminosities. My first study (Weidemann, 1967) has recently been repeated by Sion and Liebert (1977). Although the white dwarf sample was considerably enlarged, from 167 to 424 stars, the results turned out to almost exactly the same : if one restricts the method to the brighter, more complete sample, above $\log L / L_{\odot}=-3.3$, corresponding to $M_{b o l}=13$, one obtains, with cooling ages between 1 and $1.4 \cdot 10^{9}$ yrs, white dwarf birth rates, $X_{\text {wo, }}$, between 1.1 and $0.65 \cdot 10^{-12} \mathrm{pc}^{-3} \mathrm{yr}^{-1}$ which is, of course, still a lower limit. From Green's recent luminosity function for hot white dwarfs I derive $X_{\text {WD }}=1.4 \cdot 10^{-12} \mathrm{pc}^{-3} \mathrm{yr}^{-1}$. From Eggen and Bessell's (1978) study at the south galacti'c pole $\left(0.3 \cdot 10^{-3} \mathrm{WD} / \mathrm{pc}{ }^{3}\right.$ down to $\mathrm{b}-\mathrm{y}=0.05$, corresponding to $\mathrm{T}_{\text {eff }}=18000 \mathrm{~K}, \log \mathrm{L} / \mathrm{L}_{\odot}=-1.8$, $\left.\log t_{c 00 c}=8.1\right)$ I obtain $2.4 \cdot 10^{-12} \mathrm{pc}^{-3} \mathrm{yr}^{-1}$. From his proper motion studies Luyten (1975) has carefully estimated the frequency of white dwarfs in space. He predicts 27000 WD's within $100 \mathrm{pc}$, down to color class $k$, or $M_{\text {bol }} \approx 15$. With a cooling age of $4 \cdot 10^{9}$ yrs this 
corresponds to $X_{W D}=1.5 \cdot 10^{-12} \mathrm{pc}^{-3} \mathrm{yr}^{-1}$. Finally I should mention that from a comparison with planetary nebula statistics Smith jr. (1976) considered the white dwarf birth rate to be grossly underestimated. However, as I have shown subsequently (Weidemann, 1977b), this conclusion is not justified. With planetary nebula data revised by a changed distance scale one arrives again at a roughly one-to-one relation between planetary nebula and white dwarf birth rates. In summary I still propose to use $\chi_{w p}=2 \cdot 10^{-12} \mathrm{pc}^{-3} \mathrm{yr}^{-1}$ and I consider this figure to be established within a factor of two, i.e. comparatively better than the data derived from supernova statistics (see below).

\section{Fraction of DA stars, non-DA stars}

The nature of $D B$ and other non-DA stars, especially their origin, is still not understood. After a critical survey of existing proposals to change DA to DB stars during their cooling history I favored (Weidemann, 1975) the idea that both groups are different from the beginning. The strongest objection at that time - the nonexistence of helium-rich atmospheres above $\mathrm{T}$ eff around $20000 \mathrm{~K}$ - has recently been removed by Koester's (1979) study which showed that the DB's in fact extend up at least $30000 \mathrm{~K}$ and thus do form a normal cooling sequence. On cooling out of the DB range, below $12000 \mathrm{~K}$, these stars will appear as DC or $\lambda 4670\left(C_{2}\right)$ stars, depending on their carbon abundance. For some time it was thought that the $\lambda 4670$ stars form a separate group, kinematically, and thus may have a different origin. However, it now appears that this is not so if high velocity selection effects are taken into account (Humphreys et al., 1979). At lower temperatures there seem to be relatively more WD with hydrogen-poor atmospheres - a fact which could be understood by convective mixing (Koester, 1976, Vauclair and Reisse, 1977, D'Antona and Mazzitelli, 1979a)- however it is more difficult to recognize the hydrogen-rich objects below the range of visibility of the hydrogen lines, and stars which originally have been classified DC do now appear to be H-rich (Wickramasinghe and Bessell, 1979), see also Wehrse and Liebert (1979). From the viewpoint of origin it is important to know the ratio of white dwarfs born as DA's to those born as non-DA's. It can be roughly estimated by the fraction of $D B$ stars which Greenstein (1969) found in his sample of blue GD stars to be $17 \%$. I recently checked this figure by a comparison of space densities down to a limiting absolute magnitude of $M_{\checkmark}=11.5 \mathrm{i.e.} \mathrm{in} \mathrm{the} \mathrm{cooling} \mathrm{range} \mathrm{of}$ $D B^{\prime} s$, and obtain within $m-M=3.5$ (50pc) $40 \mathrm{DA}$ 's versus $7 \mathrm{DB}$ 's, or $15 \% \mathrm{DB}^{\prime} s$. It should be mentioned in this context, however, that the closest $D^{\prime}$ 's are at $m-M=3.2$ or 3.4 , whereas already within $m-M=2.5$ there are $20 \mathrm{DA}^{\prime} \mathrm{s}$, so the true space density of $\mathrm{DB}^{\prime} \mathrm{s}$ may be lower. Also for cluster white dwarfs we find only one DB (in the Hyades) versus at least $12 \mathrm{DA}$ 's (in the Hyades, Praesepe, and Pleiades), the polar cap study by Eggen and Bessell (1978) shows 4 non-DA stars vs. 16 DA's down to $(b-y)=0.3$ but only one DB vs. $10 \mathrm{DA}$ in the hotter range. We may check this by looking into a closer neighborhood for the cooled down $\mathrm{DB}^{\prime} \mathrm{s}$ : the DC's and $\lambda 4670$ stars which have a cooling age of about $2 \cdot 10^{9}$ years. With 4 DC's and 3 $\lambda 4670$ stars within 15 pc I derive birth rates of $2.4 \cdot 10^{-13} \mathrm{pc}^{-3} \mathrm{yr}^{-1}$, i.e. $12 \%$ of the total. In summary, we may conclude that the vast 
majority of white dwarfs cool via the DA sequence, and that constraints on general models of evolution derived from DA statistics are meaningful.

\section{Mass distribution of DA stars}

During the last decade the observational material for DA stars has been improved and enlarged to such a size that statistical investigations became meaningful. Graham's (1972) Strömgren photometry first displayed a beautiful single cooling sequence in the $(u-b)$ $(b-y)$ diagram from which a narrow distribution in surface gravities and therefore mass seemed evident. Considering only differential changes for the lower, gravity dependent branch of the sequence I estimated the surface gravities to be constant within \pm 0.15 in the logarithm (Weidemann, 1971). Quantitative analysis by Wickramasinghe and Strittmatter (1972) however showed this result not to hold over an extended color range, especially the observed sequence did not follow a line of constant gravity. Wehrse (1975) was next to investigate this question by calculation of improved model atmospheres for the cool DA range: he found the Graham sequence to follow a line of constant $g$, but for an average value of $\log g=8.3$, i.e. a factor of two higher than what had been derived for the total DA ensemble by Wickramasinghe and strittmatter (1972) or Shipman (1972) who had based his work on evaluation of multichannel photometry by Oke (1974). Since Oke's ensemble comprised mostly stars which were hotter, a change of the average surface gravity along the sequence seemed possible. On the other hand, the larger masses corresponding to $\log g=8.3$, around $0.77 \mathrm{Mv}$, agreed much better with the mass range derived from gravitational redshifts by Trimble and Greenstein (1972), 0.69 - 0.87 Mo. With many new MC observations by Greenstein (1976) and additional strömgren photometry for southern DA's (Bessell and Wickramasinghe, 1978, Wegner, 1979a) as well as with numerous parallaxes obtained by USNO during these years, Schulz in his thesis (1977b) evaluated the existing DA material in a weighted least square analysis. Again he confirmed a narrow $g$ distribution: $80 \%$ of the Graham ensemble had masses between 0.40 and $0.75 \mu_{\odot}$, whereas from the radius distribution and the mass-radius relation he found 808 in the range from 0.4 to $0.9 \mu_{0}$, but already 538 within 0.4 and $0.6 \mathcal{M}_{0}$. on the other hand the tendency to higher $g$ values for the cool DA's persisted. Suspecting this to be caused by the use of different sets of model atmospheres for the hotter and cooler DA's, the Kiel group decided to construct a new set of gravity dependent atmospheric models with a higher degree of sophistication which at the same time cover the whole DA temperature range. Schulz's method of evaluation of MC data - the use of 8 broad band passes instead of Greenstein's monochromatic color indices - reduced the scatter of the derived g-values considerably and put the cool DA's again on a line of constant gravity, this time around $\log g=8$, i.e. the value which always had resulted from spectroscopic analysis for the hotter DA's (Koester, Schulz, Weidemann, 1979) (KSW). This result was also supported by Green's (1977) Strömgren photometry as well as by the average location of the Bessell-Wickramasinghe stars. Whereas Bessell and wickramasinghe (1978) concluded that the g-scatter was larger than previously supposed - partly since they enlarged their 
ensemble by simple transformations of Greenstein multichannel - to Strömgren-indices -, Wegner (1979a), in publishing his final data, after some reobservations, now also confirms that the distribution is fairly narrow and can be fitted by a Gaussian with $\sigma(\Delta \log g)=$ \pm 0.24 , corresponding to $\pm 0.15 \mu_{\odot}$. In our Kiel study we furthermore evaluated the information on $\mathrm{g}$ contained in the Strömgren $\mathrm{v}$ band which Schulz (1978) had shown to be valuable as g discriminator for the hotter DA's. Again a very narrow sequence appeared in the $(u-b)(v-y)$ diagram (see $\mathrm{KSW}$ ). Some mass distributions from $\mathrm{kSW}$ are shown in Fig. 3 .

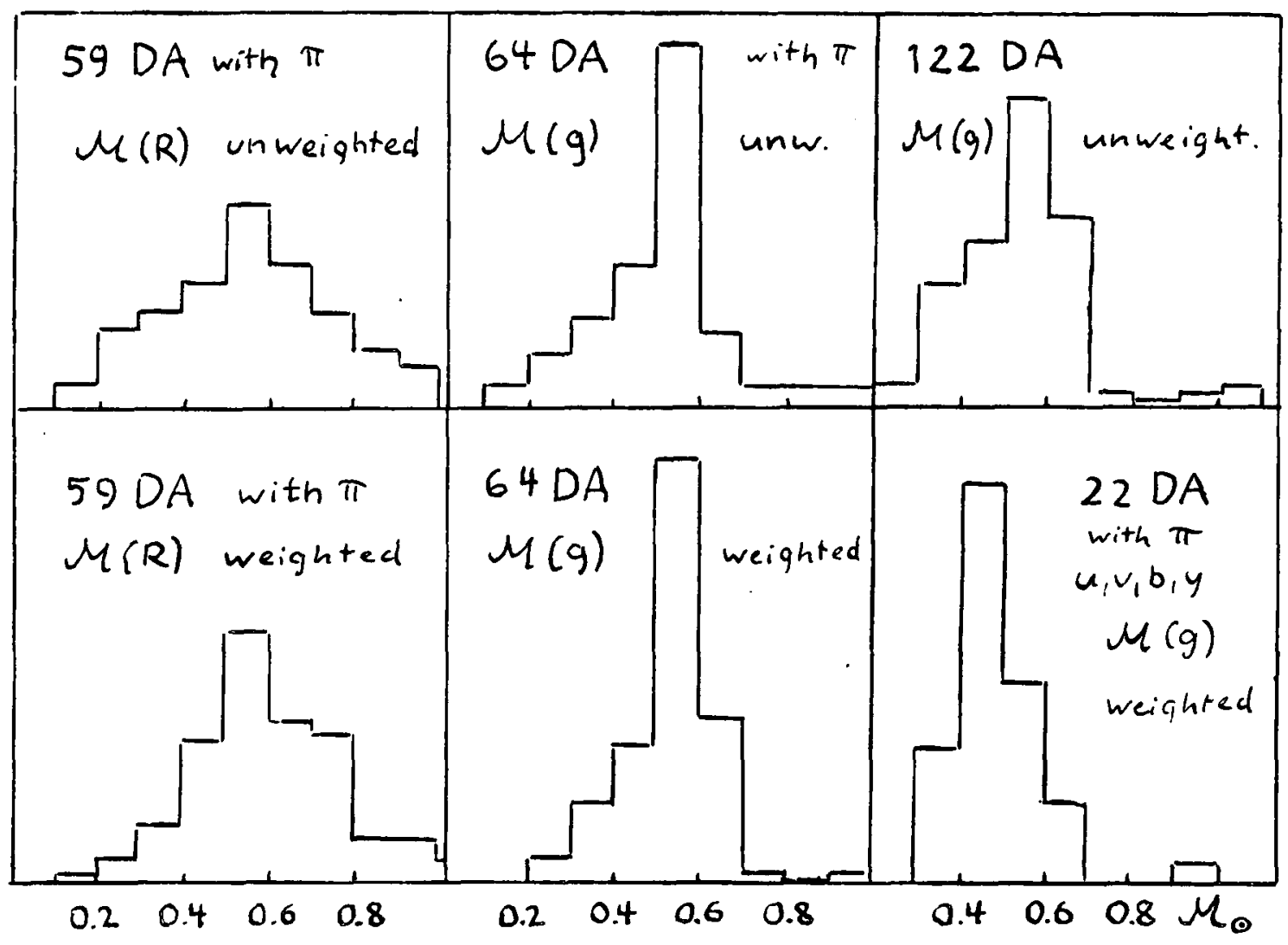

Fig.3 Mass distribution of DA white dwarfs, according to Koester, Schulz and Weidemann(1979), determined either from radii,M(R), or surface gravity, $\mathcal{M}(g)$, and mass-radius relation, for different ensembles. Stars with parallaxes, $\pi$, in general better observed. Upper row: unweighted results, lower row: weighted according to reliabilty of results for single stars.

At the same time Shipman (1979) also reanalysed DA data. Using radii only he obtained a somewhat wider mass distribution reflecting the parallax uncertainties. The mean radius, derived from the raw data, corresponds to a mass of $0.55 \mu_{\odot}(0.58$ for $\mathrm{KSW})$, however, he thinks that selection effects (favoring the detection of larger radius or smaller mass stars) could be so large as to raise the true value to $0.75 \mathcal{M}_{\odot}$. This is probably by far an overestimate, since we cannot find any significant differences in spectroscopically determined surface gravities between a close ensemble (43 DA's within $25 \mathrm{pc}$, unweighted median $\log \mathrm{g}=7.94$ ) and the more distant 
stars ( $87 \mathrm{DA}$ 's beyond $25 \mathrm{pc}, \log \mathrm{g}=7.92$ ). But even if the selection effect were operative - as stated by Shipman and Sass (1979) - it cannot reduce the discrepancy between spectroscopically derived and redshift masses, which still appear to be larger by about $0.15 \mathcal{M}_{3}$ according to newer redshift observations by Wegner $(1974,1978)$ and Greenstein et al. (1977). Although asymmetries discussed by schulz (1977.a) can possibly be made responsible for part of the redshift discrepancy this problem is not yet solved. In this context we (KSW) pointed out that a much larger mean value can hardly be reconciled with the fact that spectroscopically the DA stars are very similar to 40 Eri B - such as to exclude gravity differences larger than by a factor of 2.5 for the average, which corresponds, with $\mathcal{M}(40$ Eri B) $=$ $(0.43 \pm 0.02$ ) (Heintz, 1974) and $\log g=7.7$ to $\langle\log g\rangle$ (DA) <

$\leqslant 8.1$, or $0.64 M_{0}$. The spectroscopically derived mean values depend to a certain degree on calibration uncertainties: if the newer Tüg et al. (1977) Vega calibration had been used instead of Hayes/ Latham (1975) the mean masses derived from $g$ analysis would change from 0.52 to 0.63 in the $\mathrm{kSW}$ study. However if derived from radii the change is smaller, from 0.58 to $0.60 \mu_{\mathcal{O}}$.

In summary: although the question of the true mean value of DA masses is not yet finally settled, $0.6 \mathrm{M}_{\circ}$ seems to be the best value. The mass distribution is very narrow, with $2 / 3$ of the stars definitely within $\pm 0.15 \mu_{0}$, more probably even within $\pm 0.10 \mu_{0}$ of the mean, according to KSW (1979).

In view of the evolutionary consequences of these facts it seems especially interesting to establish the existence and/or shape of the tails of the distribution. Are there any DA's with masses, say below $0.4 M_{0}$ ? With other words we should try to narrow down observational uncertainties especially for those stars which seem to lie on the fringe of the distribution, outside the main body of stars. A first attempt in that direction has been made by Schulz and Wegner (1978) who observed stars which lay outside in the strömgren two-color diagram. They claimed to have confirmation for higher masses in two cases, still under investigation. On the other hand, a recent study of IDS observations made with the $3.6 \mathrm{~m}$ ESO telescope showed that the surface gravity derived from line profiles for 6 out of 7 stars selected for abnormal properties (higher or lower photometric gravity) falls in fact into the range $7.8 \leqslant \log g \leqslant 8$, very close to the mean value for the DA's, the only exception being an sdo (Weidemann and Koester, 1979).

\section{Population characteristics}

That white dwarfs belong to an intermediate or old stellar population has been stated several times. The velocity distribution was investigated especially by Greenstein (1969 and 1976), Wegner (1974) and Sion and Liebert (1977). The mean tangential velocity is around $40 \mathrm{~km} / \mathrm{s}$; true high velocity objects of population II are rare, at most 3\% (Schmidt 1975, Greenstein 1976). There are only minor differences between $D A$ and non-DA stars, if at all; in the direction of lower velocities for the non-DA stars. Investigations were generally based on absolute magnitudes derived from parallax- 
calibrated color-magnitude relations; otherwise the numbers would have been too small for statistical conclusions. We now have repeated these studies with individual spectroscopic parallaxes for the $140 \mathrm{DA}$ stars in our $\mathrm{KSW}$ ensemble and reproduce in essence the earlier results, although our mean color-magnitude relation deviates from that of Sion and Liebert (1977). However we could more clearly show the influence of selection favoring higher velocity objects for faint stars by comparison with a subensemble of 45 stars within 25 pc. Since this ensemble should be nearly complete, we should use it for further conclusions (Fig. 4). The median tangential velocity is $37 \mathrm{~km} / \mathrm{s}$. Similarly true space velocities for 59 DA stars with individually redshift-corrected radial velocities, as well as $\mathrm{U}, \mathrm{V}, \mathrm{W}$ velocities, have been investigated, the main result being that there is no temperature dependence of the mean values over the $D A$ range down to $T$ eff $=7000 \mathrm{~K}$. This means that $\mathrm{DA}$ stars are born essentially with the same velocity distribution which they still have after a cooling time of $10^{9}$ years. We might thus use the observed distributions directly to conclude about the progenitor population. The $|W|$ - distribution (Fig. 5) is such as to exclude a large fraction of young population $I$, - i.e. more massive progenitors. From Fig. 4 and 5 we estimate the fraction of progenitor stars more massive than $1.5 \mathcal{M}_{\theta}$ (which have $|\mathrm{w}|<.10 \mathrm{~km} / \mathrm{s}$, and $|\mathrm{z}|<170 \mathrm{pc}$ )) to be below $50 \%$.

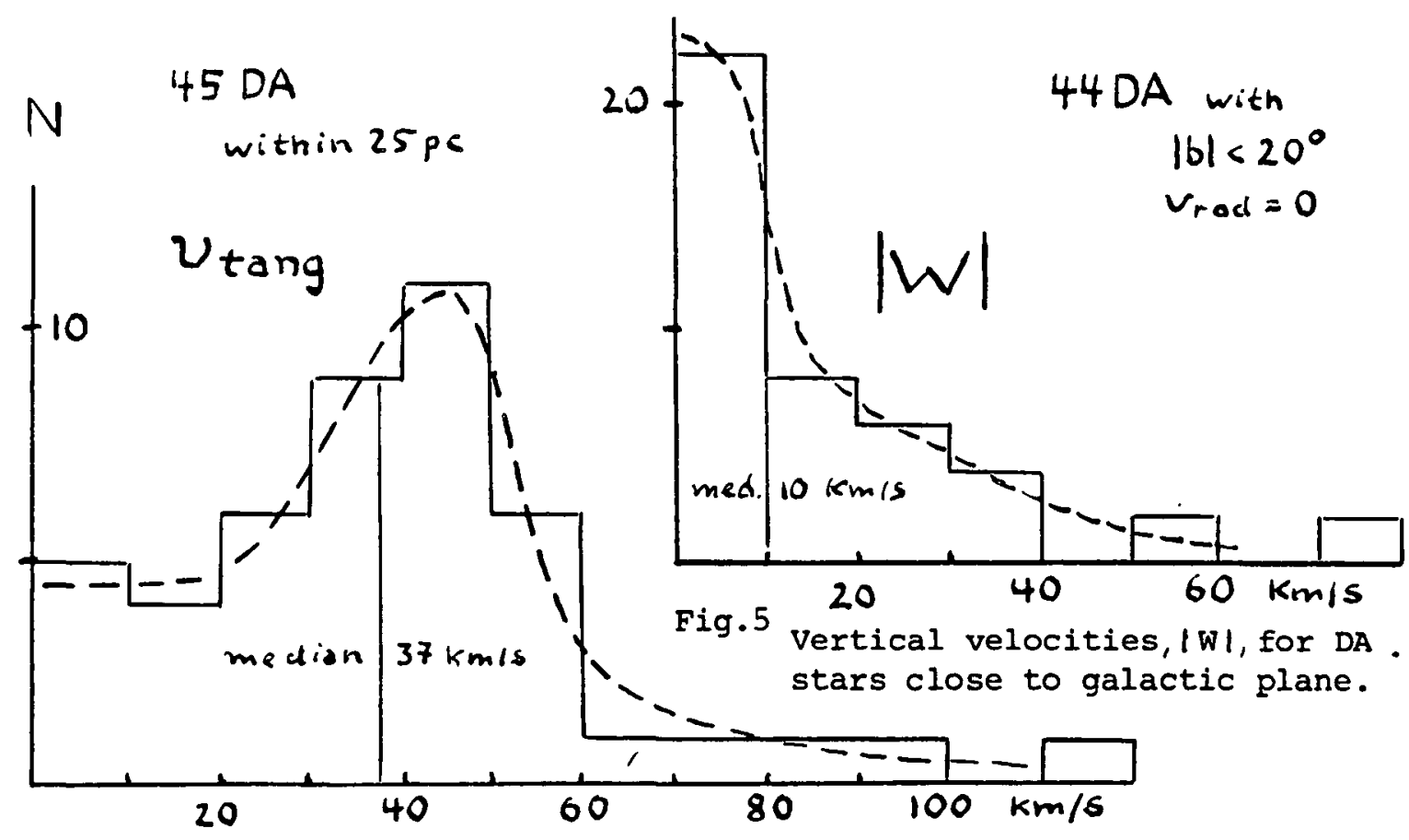

Fig.4 Distribution of tangential velocities, $v_{\text {tang }}$, for representative
ensemble of DA white dwarfs. We also evaluated the $w$ dispersion, $\sigma_{w}=19 \mathrm{~km} / \mathrm{s}$-and wielen's (1977) relation between $\sigma_{w}$ and age - to obtain a total population age of $3.5 \cdot 10^{9} \mathrm{yrs}$. Subtraction of $0.5 \cdot 10^{9} \mathrm{yrs}$. for the average DA cooling age then gives $3 \cdot 10^{9}$ yrs for the total nuclear age, which corresponds to a typical progenitor mass of $1.4 \mu_{0}$. This provides 
another constraint on models of evolution which we have used in our pilot study (Koester and Weidemann, 1979): more than 508 of the progenitors of DA white dwarfs must have masses below $1.5 \mu_{\odot}$. Furthermore we could use the space distribution. The essential parameter here is the thickness of the white dwarf layer in the galactic disk, which is unfortunately still not well determined. Oort (1958) gives $\langle|z|\rangle=220 \mathrm{pc}$, whereas Green (1977) recently derived $270 \mathrm{pc}$. Eggen and Bessell (1978) did not find blue white dwarfs beyond about 100 pc towards the south galactic pole although their survey should have reached to at least $200 \mathrm{pc}$. They consider the possibility that the hottest white dwarfs, less than $10^{8}$ years old, are strongly confined to a narrow region near the galactic plane. However they only observed stars from the GD lists which probably are very incomplete at larger distances. This situation should be clarified in a few years if studies of faint blue objects at high galactic latitudes as those by Sandage and Luyten (1967) (see also Steppe, 1978) or Luyten (1975) are extended to fainter limits. Finally, we might use knowledge about the space and velocity distribution of planetary nebulae - which show us the birthplaces of white dwarfs out to much larger distances - to reach conclusions about the progenitor population. The data - which I discussed elsewhere (Weidemann, 1977b, Koester and Weidemann, 1979) - are such as to again exclude more than 508 progenitors with masses larger than $1.5 M_{0}$

\section{Planetary Nebulae}

There seems to be no doubt that the nuclei or central stars of planetary nebulae (NPN) are immediate pre-white dwarf stages of evolution. However, more difficult to answer is the question if all white dwarfs are born in this way. Of course we know that there are white dwarfs which were produced in binary evolution with mass transfer and mass loss occuring in other evolutionary stages probably without a PN event. Furthermore, we expect that low mass horizontal branch or RR Lyrae stars of both populations do not evolve into a second giant branch and thus should become white dwarfs without a PN event (see Sweigart et al. 1974, Weidemann, 1975) but these stars are unimportant, statistically, in the evolution of the galactic disk. If we assume that the majority of NPN's are single stars (see, however Bond, 1979), i.e. if we exclude for the moment binaries, we compare the PN birth rates with those of WD's. Whereas the estimates of the FD birth rate remained nearly constant through the last decade, the PN figures have been changed several times. I have discussed this in a recent paper (Weidemann, 1977b) and shall here repeat only that the essential uncertainty for PN data arises from the uncertainty of the distances. The calculated birth rates depend on the fourth power of the distance scale factor and the latter is still debated. Similarly the existence of the so-called Harman-Seaton sequence in the HR-diagram, and especially the high luminosities derived from HeII

Zanstra temperatures have recently been questioned. However, if we use a distance scale which is intermediate between those of seaton (1968) and Cudworth (1974) one arrives again at a PN birth rate 
which is equal to that of white dwarfs within the uncertainty limits, a conclusion which had already been reached in other ways by Cahn and Kaler (1971).

If there were really equality we would conclude that all stars above the galactic turn-off, i.e. with progenitor masses above $\approx 1 \mathcal{M}_{0}$ up to $\mathcal{H}_{\text {wO }}$, must go through the PN stage, and until there is evidence against it, we shall use this as a working hypothesis. I should mention in this context that the evolutionary scheme proposed and discussed by wood and Cahn (1977) and Cahn and Wyatt (1978) which evaluates information about the Mira period-distribution - with Miras again considered as immediate progenitors of PN stages - leads to

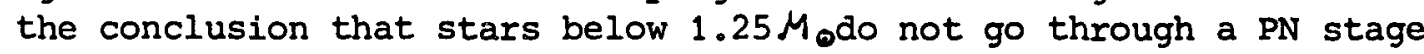
but evolve directly into white dwarfs by steady mass loss. Similarly, calculations of evolution with steady mass loss according to the Reimers formula, through the double-shell burning stage into the white dwarf region - by Schönberner (1979) in Kiel - indicate that stars below $1.4 \mathcal{M}_{\mathcal{O}}$ may lose their envelopes by stellar winds only. However, the mass loss rates at the tip of the second giant branch reach such high values $\left(10^{-6} \mathcal{M}_{\odot} y^{-1}\right)$ as to approach an unsteady $\mathrm{PN}$ ejection. These questions must be further investigated. Within our working hypothesis, we can understand the narrow mass distribution of DA white dwarfs as being caused by unsteady PN mass loss in the final stages of double-shell burning which occurs at a critical luminosity (see Fig. 3 in Weidemann, 1975). We can then predict back the mass distribution of central stars of PN - it should be similarly narrow as that of the DA's around $0.6 M_{0}$. By the core-mass luminosity relation (Paczynski, 1970) we thus predict also the HR diagram position for the majority of NPN, around $\log \mathrm{L} / \mathrm{L}_{0}=3.5$.

This conclusion does not favor the recent scheme for PN evolution proposed by Renzini (1979), according to which the hotter NPN with lower luminosities are more massive than those at the cooler end of the NPN range. Evidently the NPN which become normal WD's with H $1 \approx 0.6 M_{\odot}$ should also pass this region, and these are much more numerous than those with higher masses.

If the DA white dwarfs are preceded by mainly low mass, doubleshell burning stars one might use qualitative arguments or quantitative results to estimate the remnant unburned helium and hydrogen envelope masses. D'Antona and Mazzitelli (1979a and b) have argued that the remaining helium mass must be of the order of the maximum mass allowed for burning not to occur, i.e. around $2 \cdot 10^{-2} \mathrm{~J} / 0$ for degenerate $\mathrm{c} / \mathrm{O}$ core masses of $0.6 / \%$, since helium shell burning will not cease before this value is reached and mass loss cannot reduce the remaining envelope afterwards due to the short evolutionary time scales in these pre-white dwarf stages. Schönberner (1979) on the other hand, finds that helium shell flashes which lead to mixing of hydrogen and excursions to the red giant region may still occur on the white dwarf branch for $\mathrm{T}_{\text {off }}>10^{\mathrm{g}} \mathrm{K}$, around $10^{2} \mathrm{~L}_{0}$, where the helium envelope mass is already below $2 \cdot 10^{-2} \mu_{0}$, - an event which may 
be visible in the case of FG Sagittae. However in no case could the total hydrogen envelope be removed by steady mass loss such as to obtain He stars or DB progenitors.

The question of the progenitor mass range of PN can also be attacked in other ways. Kaler, Iben and Becker (1978) have tried to explain the enhancements of $\mathrm{He} / \mathrm{H}$ and $\mathrm{N} / \mathrm{O}$ ratios in $\mathrm{PN}$ by surface enrichments due to dredge-up processes during the preceding evolution. They conclude that the progenitor masses for PN with the highest observed enhancements must be larger than $3-5 M_{0}$. Tuchman, Sack and Barkat (1978) considered Mira stages and PN ejection due to pulsational instabilities and find that PN formation is possible at least up to $6 M_{0}$. Mass loss preceding PN ejection can be considerrable, as estimated by Capriotti (1978) in his investigation of giant halos around PN. PN of type $I$, in the classification of Peimbert (1978) have higher He and $\mathrm{N}$ abundances, are kinematically population $I$, and show extremely filamentary structure, or morphological irregularities indicative of larger masses $\left(0.8 M_{\odot}\right.$ in the case of NGC 2818). We thus conclude that white dwarfs born from PN occur out of a wide range of progenitor masses, from a lower limit, probably close to the galactic turn-off up to at least $6 / 1_{0}$.

Relation between initial and final masses and limiting mass for white dwarf production

Our evolutionary scheme is very dependent on the initial/final mass relation, as discussed in the introduction, which is determined by the total mass loss towards the white dwarf stage. There are strong reasons to assume that $\mathcal{M}_{f}\left(\mathcal{M}_{i}\right)$ is not a single-valued function of the initial mass, but that differential mass loss occurs, depending e.g. on angular momentum, magnetic fields, composition etc. Still we might take the evidence of a comparatively narrow DA mass range as an indication that such an approximation is not bad, - with other words, that even if there is differential mass loss during stellar evolution the final outcome is very similar. I have tried to understand this by some kind of self-regulating mechanism (see Weidemann, 1977a). What do we know empirically about progenitor masses? There are at least two possibilities. First, we might use white dwarfs in wide binaries, if we can estimate the total age of the system from the companion, and by subtraction of the cooling age of the WD obtain the evolutionary age of the progenitor, and thus its mass. This method has been applied by wegner $(1973,1979 \mathrm{~b})$ but is in practice not very helpful since there are few companions for which an evolutionary age can be determined. More powerful is the second method, based on white dwarfs in open clusters with known turn-off ages. It has been used by especially by Sweeney (1976) whose results indicated considerable mass loss and white dwarf production up to at least $4 M_{0}$ or higher. Her data were very helpful in my discussion of possible $\mathcal{M}_{\mathcal{H}}\left(\mathcal{M}_{i}\right)$-relations as displayed in Fig. 1 of my paper (Weidemann, 1977a). Do we have new information in the meantime? Yes: the investigation by Romanishin and Angel (1979) which demonstrates that white dwarfs are produced probably even beyond 
the former upper limit of $\mu_{i}=6 \mu_{0}$ - which was based on the single white dwarf in the Pleiades. According to the KSW analysis, the Pleiad is a normal $D A$ with a slightly larger than average mass $\mu(g)=0.73 \mu_{0}, \mu_{(R)} \equiv 0.91 \mu_{0}$, whereas the Hyades white dwarfs with progenitor masses from 2.5 to $4 \mu_{\sigma}$ (Weidemann, 1977a) - have masses $\mu_{f}$ between 0.50 and $0.69 M_{0}, i . e$. are comparatively smaller. The $\mathcal{M}_{f}\left(\mathcal{M}_{i}\right)$ relation, accordingly, should be very flat between the turn-off mass, $\approx 1 M_{0}$ and at least $3 H_{0}$. Will it bend upwards to the Chandrasekhar limit sharply beyond $6 M_{\odot}$ ? This depends on the value of $\mathcal{H}_{w D}$, one of the most discussed parameter in the literature (e.g. Tinsley 1979) in connection with supernova rates, element production, and pulsar statistics. Tinsley (1979) most recently assumes that stars die as white dwarfs if $\mu_{1}<4 \mu_{0}$ as supernovae type I from 4 to $6.5 \mathrm{Mo}$ and as SN II above $6.5 \mathrm{M}_{\mathrm{o}}$. Pulsar statistics, on the other hand, still favors higher birth rates, and thus lower values of $\mu_{\text {wo }}$. After reviewing the existing results $I$ came to the conclusion that for the time being it is not advisable to use supernova or pulsar statistics in this context, since the predicted rates vary between $1 \mathrm{SN}$ in the Galaxy in 4 years (Lande and Stephens, 1977), or $1 / 6$ to $1 / 40$ yrs, depending on the electron density of the interstellar medium (Taylor and Manchester, 1977), to 1 SN II/11 yrs (Tammann, 1977) or 1/80 yrs for SN remnants (Caswell and Lerche, 1979). If the SN/WD birth rate were known we could of course use it to discriminate between models within our evolutionary scheme (see Koester and Weidemann, 1979), at present, however, we have to await better data.

From a theoretical point of view one is inclined to think that the final fate of a star is decided by the way carbon ignition occurs or is avoided in the late double-shell burning phases. If Mengel (1976) is correct, the very fact that a cool degenerate C/0-core develops causes the high luminosity which - acting like a valve-terminates the nuclear evolution by increased mass loss. If so we should expect that in order to reach the critical core-mass for collapse and supernova explosion, one has to avoid a degenerate core, i.e. go to masses $\mathcal{M}_{i}$ above $7 \mathcal{M}_{\odot}$, where carbon ignites in a nondegenerate core (Paczynski, 1970). It also may be that the decisive event for the dichotomy is carbon ignition in a shell (Iben, 1972). At any rate it may be a very small transition range in the temperature-density evolution which separates the two classes of star deaths. With recent evidence from $X$-ray binaries and the binary pulsar, neutron star masses seem to be confined to a small mass range also, just above the Chandrasekhar limit (Weaver and woosley, 1979, Taylor et al. 1979). We thus may finally remember as a first approximation a very simple $\mu_{f}\left(\mathcal{H}_{i}\right)$ relation which is nearly a two-step function, with $\mu_{f} \approx 0.6 \mu_{0}$ below $\mu_{w D}$ and $\approx 1.5 \mu_{0}$ above. The value of $\mu_{w_{D}}$ itself is somewhat more problematic. We have to distinguish the fact that there are evidently some cases in which stars up to $7 M_{0}$ produce white dwaxfs from what happens in the average. I have given reasons (Weidemann, 1977a) why $\mu_{\text {wD }}$ should not be considered to be a strict limit. Until we know it better we should thus be careful in using this value for calculations of models of galactic evolution. 
I have tried to lay some groundwork on which we can base further investigations to understand the origin and distribution of white dwarfs. I could by no means be exhaustive. Especially, I did not touch the question of binary evolution which - as shown by the many contributions in this Conference - is at the focus of much interest.

It is well known (e.g. Abt and Levy, 1976) that the majority of stars belongs to binaries, with only about $30-40 \%$ of all stellar systems being single, and even these may have very faint or planetary companions. However, the white dwarf evolution scheme as presented here should still remain essentially correct, since about 508 of all binaries are wide systems in the sense that the components cannot exchange mass during their evolution. We thus expect about 708 of all stars, in the main WD progenitor mass range, to follow single star evolution. On the other hand about 308 of the systems will undergo mass exchange or interact in a way about which little is known. However I estimate that less than one percent end up as cataclysmic variables. Since in many of the closer systems white dwarfs would be unobervable we must exclude them from our ensemble. However, the true total white dwarf birth rate might be higher by about 508 - which remains within the uncertainty of $X$ wD given above.

I futhermore felt that statistical data are not sufficient as yet as to deal with magnetic white dwarfs and $\mathrm{zZ}$ Ceti stars. I finally omitted the discussion of the luminosity function at the cool end of the white dwarf sequence since new observational results are just coming in (Liebert et al.,1979).

Extrapolating from what we have learned during the last decade, and with the Space Telescope ahead, we might be very optimistic about progress in the next years. At least we are well under way. 
Abt, H.A., Levy, S.G.: 1976, Ap.J.Suppl. 30, 273

Bessell, M.S., Wickramasinghe, D.T.: 1978, Mon.Not.R.A.S. 182, 275

Bond, H.E.: 1979, this Conference

Cahn, J.H., Kaler, J.B.:1971, Ap.J.Suppl. 22, 319

Cahn, J.H., Wyatt, S.P.: 1978, Ap.J. 221, 163

Capriotti, E.R.: 1978, I.A.U. Symp. No.76, Planetary Nebulae, ed. Y. Terzian, P. 263, D. Reidel, Dordrecht

Caswell, J.I., Lerche, I.: 1979, Mon.Not.R.A.S. 187, 201

Cudworth, K.M.: 1974, Astr.J. 79, 1384

D'Antona , F., Mazzitelli, I.: 1979a, Astr.Ap. 74, 161 $1979 b$, preprint

Eggen, O.J., Bessell, M.S.: 1978, Ap.J. 226, 411

Graham, J.A.: 1972, Astron.J. 77, 144

Green, R.F。: 1977, Ph.D.Thesis, Calif.Inst.Technology

Greenstein, J.L.: 1969, Ap.J. 158, 281

Greenstein, J.L.: 1976, Astron.J. 81, 323

Greenstein, J.L., Boksenberg, A., Carswell, R., Shortridge, K.: 1977, Ap.J. 212,186

Hayes, D.S., Latham, D.W.: 1975, Ap.J. 134, 683

Heintz, W.D.: 1974, Astron.J. 79, 819

Humphreys, R.M., Liebert, J., Romanishin, W., Strittmatter, P.A.: 1979

Publ. Astron.Soc.Pac. 91, 107

Iben jr., I.: 1972, Ap.J. 178, 433

Kaler, J.B., Iben jr., I., Becker, S.A.: 1978, Ap.J. 224, L63

Koester, D.: 1976, Astron.Ap. 52, 415

Koester, D.: 1979, this Conference

Koester, D., Weidemann, V.: 1979, Astron.Ap. in press

Koester, D., Schulz, H., Weidemann, V.: 1979, Astron.Ap. in press

Lande, K., Stephens, W.E.: 1977, Astrophys.SpaceSci. 49, 169

Larson, R.B., Tinsley, B.M.: 1978, Ap.J. 219, 46

Liebert, J., Dahn, C.C., Gresham, M., Strittmatter, P.A.: 1979, Ap.J., in press

Luyten, W.J.: 1975, Proper motion survey with the 48 inch Schmidt

telescope, XL。 Univ. Minnesota

Mengel, J.G.: 1976, Astron.Ap. 48, 83

Oke, J.B.: 1974, Ap.J.Suppl. 27, 21

Oort, J.H.: 1958, Stellar Populations, North Holland Publ. Co., Amsterdan, p. 146

Paczynsk1, B.: 1970, Acta Astron. 20, 47 
Peimbert, M.: 1978, I.A.U. Symp. No. 76, Planetary Nebulae, ed. Y.Terzian, P.215, D. Reidel, Dordrecht

Reimers, D.: 1975, Mem.Roy.Sci. Liège $\underline{8}, 369$

Renzini, A.: 1979, star and Star Systems, Proc. 4th Europ. Regional Meeting Ast., Uppsala, D.Reidel, Dordrecht ,p.155

Romanishin, W., Angel, J.R.P.: 1979, in press

Sandage, A., and Layten, W.J. 1967, Ap.J., 148, 767

Schmidt, M.: 1975, Ap.J. 202, 22

Schönberner, D.: 1979, Astron.Ap. in press

Schulz, H.: 1977a, Astron.Ap. 54, 315

Schulz, H.: 1977b, Ph. D. Thesis, Univ. Kiel

Schulz, H.: 1978, Astron.Ap. 68, 75

Schulz, H., Wegner, G.: 1978, Paper presented at AAS Meeting Austin Texas

Seaton, M.J.: 1968, Astrophys. Letters 2, 55

Shipman, H.I.: 1972, Ap.J. 177, 723

Shipman, H.L.: 1979, Ap.J. 228, 240

Shipman, H.L., Sass, C.A.: 1979, Ap.J. in press

Sion, E.M., Liebert, J.: 1977, Ap.J. 213, 468

Smith jr., H.: 1976, Astron.Ap. 53, 333

Steppe, H.: 1978, Astron. Ap.Suppl. 31, 209

Sweeney, M.A.: 1976, Astron.Ap. 49.., 375

Sweigart, A.V., Mengel, J.G., Demarque, P.: 1974, Astron.Ap. 30,13

Tammann, G.: (1977), 8th Texas Symp. Rel. Ap. Ann.

N.Y.Acad. Sci. 302, p.61

Taylor, J.H., Manchester, R.N.: 1977, Ap.J. $\underline{215}, 885$

Taylor, J.H., Fowler, L.A., Mc Culloch, P.M.: 1979, Nature 277, 437

Thuan, X.T., Hart, M.H., Ostriker, J.P.: 1975, Ap.J. 201, 756

Tinsley, B.M.: 1979, Ap.J. 229, 1046

Trimble, V., and Greenstein, J.L. 1972, Ap.J., 177, 441

Tuchman, Y., Sack, N., Barkat, Z.: 1978, Ap.J. 225, L137

Tüg, H., White, N.M., Lockword, G.W.: 1977, Astron.Ap. 61, 679

Vauclair, G., Reisse, C.: 1977, Astron.Ap. 61, 415

Weaver, T.A., Woosley, S.E. 1979, 9th Texas Symp.Rel.Ap., Ann. N.Y.

Wegner, G.: 1973, Mon.Not.R.A.S. 165,271

Acad. in press

Wegner, G.: 1974, Mon.Not.R.A.S. 166,271 
Wegner, G.: 1978, Mon.Not.R.A.S. 182, 111

Wegner, G.: $1979 \mathrm{a}$, preprint

Wegner, G.: 1979b, this Conference

Wehrse, R.: 1975, Astron.Ap. 39, 169

Wehrse, R., Liebert, J.: 1979, Astron.Ap. in press

Weidemann, V.: 1967, Z.f.Astrophys. 67, 286

Weidemann, V.: 1971, I.A.U. Symp. No. 42, White Dwarfs, p.81 Ed.

W. Luyten, D. Reidel, Dordrecht

Weidemann, V.: 1975, Problems in Stellar Atmospheres and Envelopes

Ed. B. Baschek, W.H. Kegel, G. Traving,

Springer Verlag, Heidelberg, p.173

Weidemann, V.: 1977a, Astron.Ap. 59, 411

Weidemann, $V_{0}:$ 1977b, Astron.Ap. 61, L27

Weidemann, V., Koester, D.: 1979, submitted to Astron.Ap.

Wickramasinghe, D.T., Bessell, M.S.: 1979, Mon.Not.R.A.S. 186, 399

Wickramasinghe, D.T.,Strittmatter, P.A.: 1972, Mon.Not.R.A.S.160,421

Wielen, R.: 1977, Astron.Ap. 60, 263

Wood, P.R., Cahn, J.H.: 1977, Ap.J. 211, 499 